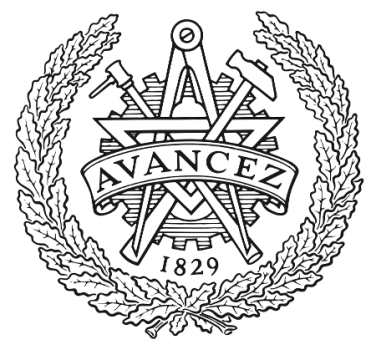

CHALMERS

UNIVERSITY OF TECHNOLOGY

\title{
Integrated calibration noise coupler for room temperature SKA band 1 feed system
}

Downloaded from: https://research.chalmers.se, 2023-04-26 15:05 UTC

Citation for the original published paper (version of record):

Flygare, J., Billade, B., Dahlgren, M. et al (2017). Integrated calibration noise coupler for room temperature SKA band 1 feed system. Proceedings of 2017 IEEE International Symposium on Antennas and Propagation \& USNC/URSI National Radio Science Meeting, APSURSI 2017, San Diego, CA, USA, 9-14 July 2017, 2017: 777-778.

http://dx.doi.org/10.1109/APUSNCURSINRSM.2017.8072431

N.B. When citing this work, cite the original published paper. 


\title{
Integrated calibration noise coupler for room temperature SKA Band 1 feed system
}

\author{
Jonas Flygare*, Bhushan Billade, Magnus Dahlgren, Bo Wästberg, Miroslav Pantaleev \\ Onsala Space Observatory, Dept. of Earth and Space Sciences \\ Chalmers University of Technology, Sweden \\ jonas.flygare@chalmers.se
}

\begin{abstract}
This paper presents design of a novel coupler for the injection of calibration signal into the RF path of the SKA Band 1 quad-ridged flared horn, covering frequencies from $350-1050$ MHz. The coupler is integrated in the feed horn and provides a coupling factor of $-35 \mathbf{d B}$. The calibration signal is injected before the first amplification stage, without any degradation in the noise performance of the room temperature system.

The design uses a mode suppression ring to damp higher order modes leaking out of the horn aperture.
\end{abstract}

\section{INTRODUCTION}

The Square Kilometre Array (SKA) is an international collaboration project to construct the world's largest and most sensitive radio telescope in its frequency range [1]. As the name suggest the total collecting area of the array will be a square kilometre in size. The science capabilities are immense and will open the door to a greatly enhanced understanding of our universe. The first phase of the SKA project will finish with 133 constructed $15 \mathrm{~m}$ offset Gregorian reflector dishes, each equipped with five feed packages, covering frequencies from $350 \mathrm{MHz}$ to $13.8 \mathrm{GHz}$.

The Band 1 feed package presented in details in [2] is based on a dual-polarized room temperature $3: 1 \mathrm{QRFH}$, see Fig. 1. covering frequencies $350-1050 \mathrm{MHz}$ and cryogenic lownoise amplifiers (LNA). Aperture efficiency is above $70 \%$ across the band on the $15 \mathrm{~m}$, offset Gregorian, dish. With return loss better than $-10 \mathrm{~dB}$ and receiver noise between 9 and $14 \mathrm{~K}$, a sensitivity average above $4.2 \mathrm{~m}^{2} / \mathrm{K}$ over the band is achieved.

For radio astronomy applications a precise gain calibration of the system is crucial to compensate for the system gain fluctuation and drift. This calibration is usually done by injecting a broadband white noise of known power spectral density, in the RF path of the system as early as possible, and then turning this noise source on and off at a certain time interval. In a cryogenic system this is usually achieved by using a micro-strip or waveguide directional coupler before the first active component. Because of the cryogenic temperature, the insertion loss of the coupler does not contribute much in the overall receiver noise. Development of the room temperature LNA with excellent noise performance [3] enabled the Band 1 re-design to a completely room temperature based system with the LNAs integrated directly in the horn ridges. This reduces the complexity of the system and is appealing considering both maintenance and operation. It is especially advantageous

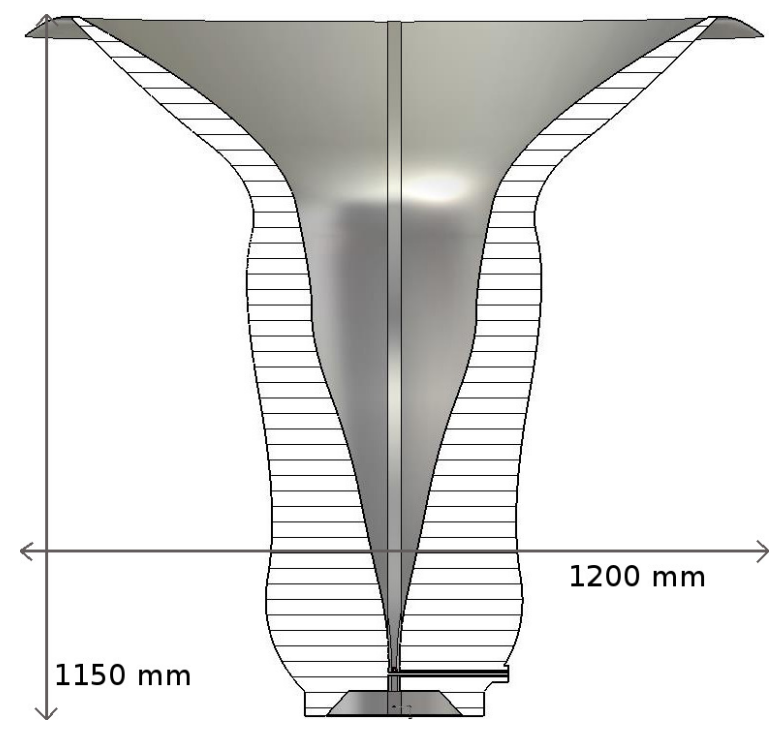

Fig. 1. Cross-section of the quad-ridge flared horn for Band 1, at the bottom the back-short cavity is seen where the noise coupler is located (see Fig. 2 for a zoomed view).

when considering a large number of telescopes, to reduce the number of cryostats and respectively the power consumption. For SKA Band 1 at room temperature, even an extremely low loss directional coupler would add significantly to the overall system noise temperature. Therefore, we can not use this technique and a different approach is needed.

In this paper we present a novel design of a noise calibration coupler for the room temperature Band 1 system, integrated in the feed horn with mode suppression technique to mitigate noise coupling to nearby telescopes.

\section{NOISE COUPLER CONCEPT}

The feeding of the horn consist of orthogonal coaxial pins exciting each pair of opposing ridges for respective polarization. The noise coupler concept is based on introducing a radiative element inside the feed that will provide an equal level of about $-35 \mathrm{~dB}$ coupling to the QRFH feeding ports, while minimizing the calibration noise radiating outside the horn. The coupler consists of a bracket-shaped probe over the back-short plane. To inject equal levels of noise for respective polarization, the coupler is placed $45^{\circ}$ relative the orthogonal 


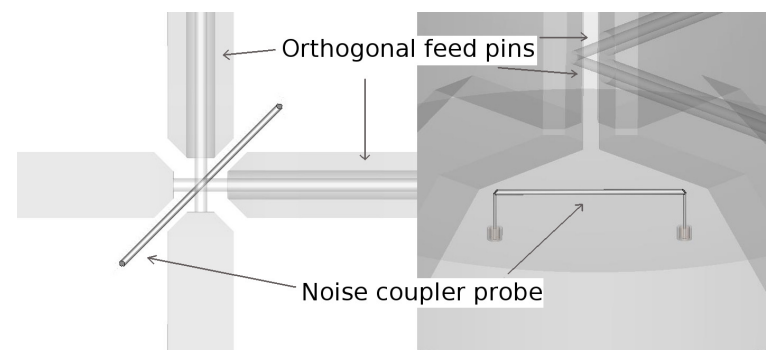

Fig. 2. Concept of the noise coupler design in the horn back-short; a bracket shaped injection probe (highlighted) in $45^{\circ}$ to the orthogonal feed pins (transparent). (Left) Top View (Right) Side View.

polarization pins as seen in Fig. 2 The SKA requirement for coupled calibration noise signal is between $5-13 \%$ of the total system noise.

\section{OpTIMIZATION}

The noise coupler horizontal pin height above the ground plane and distance between the vertical pins is optimized to couple at $-35 \mathrm{~dB}$ level with a $4-5 \mathrm{~dB}$ maximum variation over the band. A stronger coupling level is not desired as this would mean loss of incoming power into the coupler termination. The coupler is connected to a temperature stabilized noise diode through a standard SMA interface. The slope correction and power level adjustment is achieved via an equalizer and attenuator following the noise diode. The noise coupler is terminated in a $50 \Omega$ load at the coaxial output in the backshort. In Fig. 3 the return loss for the two polarizations in the horn are seen as solid lines. The corresponding dashed lines show the noise injected from the coupler to each polarization fulfilling the $-35 \mathrm{~dB}$ requirement across the band.

Since the dimension of the back-short cavity measures in the range of $\sim \lambda$ for the highest frequencies the coupler introduces excitation of higher order modes. A mode suppressor is therefore implemented and optimized in shape, thickness and placement to reduce coupling to the aperture. To minimize the unwanted loading of the polarization feed lines $(50 \Omega)$, the design is a four-piece petal ring with cutouts in $45^{\circ}$ relative the ridges, instead of a classic full ring mode suppressor [4]. The thickness of the four-piece petal is $5 \mathrm{~mm}$ to ease the implementation in the mechanical design, this could be lowered to $1 \mathrm{~mm}$ if desired without loss of suppressing function. Preferably the suppressor would reside in the back-short of the system, however this loads the feed impedance in an undesirable way. On the left side of Fig. 4 we can see a mode distribution at the aperture that clearly shows unwanted mode excitation from the coupler. On the right it is illustrated how this effect can efficiently be reduced by the mode suppressor. The four-piece petal is located near the orthogonal excitation ports, as this proved to affect the radiation performance the least for the two polarizations.

\section{CONCLUSION}

We present a novel design for noise calibration in a room temperature 3 : 1 wide-band feed system for ultra-high

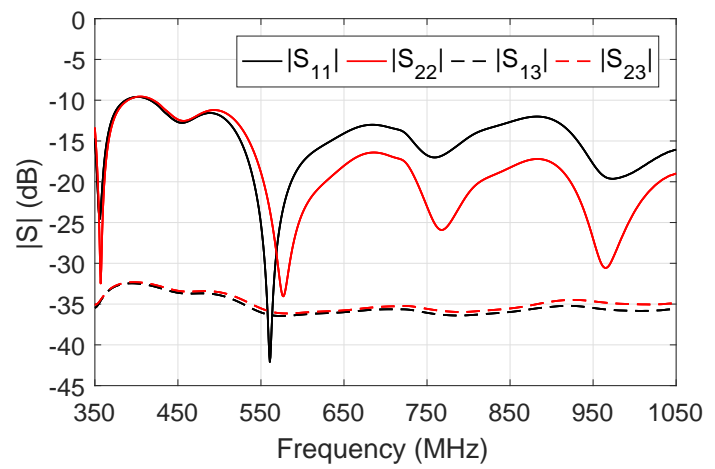

Fig. 3. Return loss and noise injection level for the horn. Noise coupler is defined as port 3 .

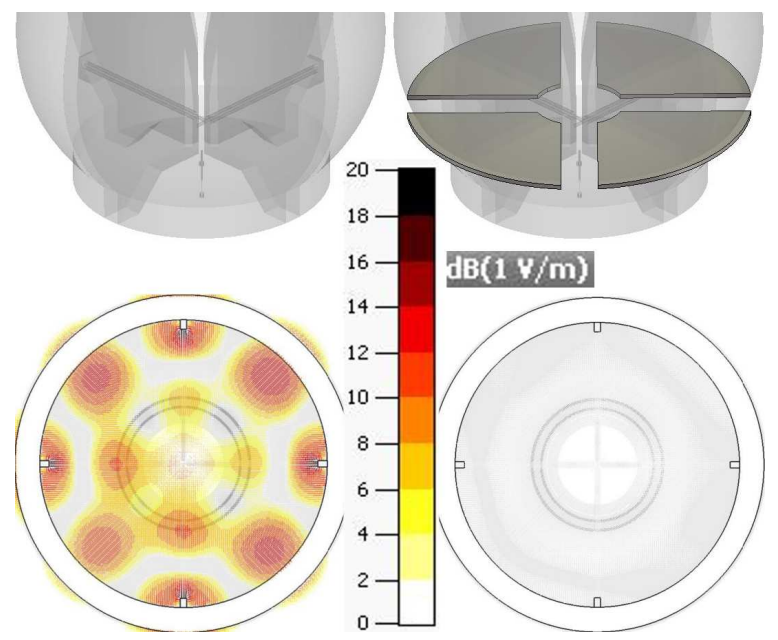

Fig. 4. Illustration of the E-field at the aperture $(800 \mathrm{MHz})$ from the noise coupler without (left) and with (right) the mode suppressor.

frequencies through an integrated noise coupler together with a tunable four-piece petal mode suppressor.

\section{ACKNOWLEDGMENT}

This work has been funded by Swedish VR Research Infrastructures Planning Grant Swedish contributions to the SKA radio-telescope in its pre-construction phase.

\section{REFERENCES}

[1] P. Dewdney, "SKA1 system baseline design," pp. 1-98, March 2013 [Online]. Available: https://www.skatelescope.org/wpcontent/uploads/2014/11/SKA-TEL-SKO-0000002-AG-BD-DD-Rev01SKA1_System_Baseline_Design.pdf

[2] B. Billade, J. Flygare, M. Dahlgren, B. Wästberg, and M. Pantaleev, "A Wide-band Feed System for SKA Band 1 Covering Frequencies From 350 - $1050 \mathrm{MHz}, "$ in Proc. 10 ${ }^{\text {in }}$ Euro. Conf. Antennas Propag. (EuCAP2016), Davos, Switzerland, April 10-15, 2016, pp. 1-3.

[3] J. Schleeh, N. Wadefalk, P. A. Nilsson, and J. Grahn, "10 K room temperature LNA for SKA band 1, "|in IEEE MTT-S Int.l Microw. Symp. Digest (IMS2016)., San Francisco, United States, May 22-27, 2016.

[4] A. Akgiray, S. Weinreb, and W. Imbriale, "Design and measurements of dual-polarized wideband constant-beamwidth quadruple-ridged flared horn," in Proc. IEEE Antennas Propag. Soc. Int. Symp. (APSURSI2011), Spokane, Washington, USA, July 3-8, pp. 1135-1138, 2011. 